TRANSACTIONS OF THE

AMERICAN MATHEMATICAL SOCIETY

Volume 352, Number 11, Pages 5013-5023

S 0002-9947(00)02393-X

Article electronically published on June 13, 2000

\title{
A COMPACTIFICATION OF A FAMILY OF DETERMINANTAL GODEAUX SURFACES
}

\author{
YONGNAM LEE
}

\begin{abstract}
In this paper, we present a geometric description of the compactification of the family of determinantal Godeaux surfaces, via the study of the bicanonical pencil and using classical Prym theory. In particular, we reduce the problem of compactifying the space of bicanonical pencils of determinantal Godeaux surfaces to the compactification of the family of twisted cubic curves in $\mathbb{P}^{3}$ with certain given tangent conditions.
\end{abstract}

\section{INTRODUCTION}

Let $\mathcal{X} \rightarrow \Delta$ be a flat family of projective surfaces over a small disk in $\mathbb{C}$. Assume that the general fiber $X_{t}$ is a minimal projective surface of general type for $t \in \Delta^{*}$. Then an interesting and difficult question is how to find a distinguished canonical model for the central fiber after a possible base change, and to classify such central fibers. There are two main difficulties in studying this problem compared to a case of curves. One difficulty is that, in general, we cannot obtain a semistable reduction while preserving relative ampleness. The other difficulty is that the moduli space, $\mathcal{M}_{K^{2}, \chi}$, is unknown and the number of components is also unknown.

We study the special case in which the general fiber $X_{t}$ is a numerical Godeaux surface, namely, those with

$$
\chi\left(\mathcal{O}_{X}\right)=1 \text { and } K^{2}=1,
$$

for $t \in \Delta^{*} \cdot \chi\left(\mathcal{O}_{X}\right)=1$ and $K^{2}=1$ imply that $p_{g}=q=0$ via the construction of the cyclic unramified covering and some inequalities. The reason for choosing this kind of surface for our study is that, if $\chi\left(\mathcal{O}_{X_{t}}\right)$ and $K_{X_{t}}^{2}$ are as small as possible, then the quadratic polynomial $h^{0}\left(X_{t}, m K_{X_{t}}\right)=P_{X_{t}}(m)$ increases slower than other cases. Also, if we consider the cohomology of the holomorphic tangent bundle, which is related with the first order deformation space of $X_{t}$, we have

$$
-\chi\left(T_{X_{t}}\right)=10 \chi\left(\mathcal{O}_{X_{t}}\right)-2 K_{X_{t}}^{2}
$$

Furthermore by using Bogomolov's lemma $\left(h^{0}\left(\Omega_{X_{t}}\left(-m K_{X_{t}}\right)\right)=0\right.$ if $\left.m \geq 1\right)$ and $4 K_{X_{t}}$ 's base point freeness, then $h^{2}\left(T_{X_{t}}\right) \leq 14 K_{X_{t}}^{2}$ (cf. [C2, $\left.\S 5\right]$ ). So

$$
h^{1}\left(T_{X_{t}}\right) \leq 10 \chi\left(\mathcal{O}_{X_{t}}\right)+12 K_{X_{t}}^{2} .
$$

Received by the editors November 30, 1997 and, in revised form, March 29, 1998.

2000 Mathematics Subject Classification. Primary 14J10, 14J29.

The author would like to express his appreciation to Professor Herb Clemens for bringing his attention to this work, and for the valuable suggestions that made it possible. Also he would like to thank the referee for some comments. This work is part of a Ph.D. thesis submitted to the University of Utah in 1997. It was partially supported by the Korea Institute for Advanced Study. 
In this paper, we study such degenerations for a special subfamily of Godeaux surfaces (i.e. quotients of a quintic in $\mathbb{P}^{3}$ under a free $\mathbb{Z}_{5}$-action). In the 8dimensional family of Godeaux surfaces, there is a 4-dimensional subfamily for which the quintic is symmetric determinantal. We call these surfaces determinantal Godeaux surfaces. They were first studied systematically by Catanese in [C1]. This subfamily and its degenerations are the main object of our study. The purpose of this paper is to present a geometric description of a compactification of the family of determinantal Godeaux surfaces by studying its bicanonical pencil using classical Prym theory. The outline of our approach is as follows.

A Godeaux surface $X$ has a bicanonical pencil, this determines a rational curve $\mathbb{P}_{X}^{1}$ in $\overline{\mathcal{M}_{4}}$, the Deligne-Mumford compactification of the moduli space of genus four curves. Let $\tau$ be the generators of the fundamental group of a Godeaux surface $X$. Then $H^{0}\left(K_{X}+i \tau\right)$ for $i=1, \ldots, 4$ contains a unique curve $C_{i}$ of genus two and $C_{i}, C_{j}$ intersect transversally at one point $P\left(C_{i}, C_{j}\right)$. Denote

$$
\begin{aligned}
P_{1}=P\left(C_{1}, C_{2}\right), & P_{2}=P\left(C_{1}, C_{3}\right), \\
P_{3}=P\left(C_{4}, C_{2}\right), & P_{4}=P\left(C_{4}, C_{3}\right), \\
P & =P\left(C_{1}, C_{4}\right), \quad Q=P\left(C_{2}, C_{3}\right) .
\end{aligned}
$$

Then $P_{1} \ldots P_{4}$ are base points of $\left|2 K_{X}\right|$ and $P, Q$ are base points of $\left|3 K_{X}\right|$. The canonical sheaf $K_{C}$ of each member $C$ in $\left|2 K_{X}\right|$ can be given as the combination of $P_{1} \ldots P_{4}$, and the two $g_{d}^{r}$ 's on $C, r=1, d=3$ (which we write $g_{3}^{1}, h_{3}^{1}$ with $g_{3}^{1}+h_{3}^{1}=K_{C}$ ) are also given in terms of these four points. Because these points are base points of the bicanonical pencil, $g_{3}^{1}$ and $h_{3}^{1}$ are both monodromy invariant. The general member $C$ in the bicanonical pencil of a determinantal Godeaux surface $X$ has an invariant unbranched double cover $\tilde{C}$ induced from a distinguished double cover of $X$. So determinantal Godeaux surface $X$ determines two rational curves $\mathbb{P}_{X, g_{3}^{1}}^{1}, \mathbb{P}_{X, h_{3}^{1}}^{1}$ in $\overline{\mathcal{R}_{7}} \times \overline{\mathcal{M}_{4}} \mathcal{W}_{3}^{1}$ where $\overline{\mathcal{R}_{7}}$ is the compactification of the space of unbranched double covers of curves of genus four, and $\mathcal{W}_{3}^{1}$ represents the family of the linear system of degree 3 with projective dimension one on curves of genus four. The Prym construction of Donagi gives a birational morphism $\Phi$,

$$
\begin{aligned}
& \mathbb{P}_{X, g_{3}^{1}}^{1} \subseteq \overline{\mathcal{R}_{7}} \times \overline{\mathcal{M}_{4}} \mathcal{W}_{3}^{1} \supseteq \mathbb{P}_{X, h_{3}^{1}}^{1} \\
& \Phi \downarrow \\
& \mathbb{P}_{X, g_{4}^{1}}^{1} \subseteq P i c_{3}^{4} \supseteq \mathbb{P}_{X, h_{4}^{1}}^{1}
\end{aligned}
$$

where $P i c_{3}^{4}$ is the family of degree 4 line bundles on curves of genus 3 . Let $\tilde{X}$ be the blow up of $X$ at the base points of $\left|2 K_{X}\right|$ and $\left|3 K_{X}\right|$. Then the geometry of $\Phi^{-1}$ allows us to describe the image of mapping

$$
\tilde{X} \stackrel{\left|2 K_{X}\right| \times\left|3 K_{X}\right|}{\longrightarrow} \mathbb{P}^{1} \times \mathbb{P}^{3}
$$

as the pull back surface induced by a degree 3 map

$$
\mathbb{P}^{1} \rightarrow \mathbb{P}\left(H^{0}(\text { Segre cubic, } \mathcal{O}(2))\right)
$$

passing through two fixed points with fixed skew tangent direction. Thus the degenerations of the surfaces are given by two possible degenerations of the corresponding twisted cubics.

It is hoped that further study will lead to similar geometric conclusions for other families of numerical Godeaux surfaces. 


\section{The family of Godeaux Curves}

Let $(x, y, z, w)$ be the coordinates of $\mathbb{P}^{3}$, and consider a natural $\mathbb{Z}_{5}$-action on $\mathbb{P}^{3}$ via

$$
(x, y, z, w) \rightarrow\left(\epsilon x, \epsilon^{2} y, \epsilon^{3} z, \epsilon^{4} w\right)
$$

where $\epsilon$ is the primitive 5 -th root of unity. The $\mathbb{Z}_{5}$-invariant quintics form an irreducible 8-dimensional family $\mathcal{M}[\mathrm{Mi}]$ :

$$
\begin{aligned}
x^{5}+y^{5}+z^{5}+w^{5} & +t_{1} x^{3} z w+t_{2} x y w^{3}+t_{3} x y^{3} z+t_{4} y z^{3} w \\
& +t_{5} x^{2} y z^{2}+t_{6} y^{2} z w^{2}+t_{7} x^{2} y^{2} w+t_{8} x z^{2} w^{2} .
\end{aligned}
$$

Since the four fixed points of $\mathbb{Z}_{5}$-action are not in $\Sigma$, the quotient $X$ is a smooth projective surface of general type, with $p_{g}=q=0, K^{2}=1$, and $\pi_{1}(X)=\mathbb{Z}_{5}$. We will denote these surfaces as Godeaux surfaces. For a Godeaux surface, the bicanonical pencil (resp. the tricanonical linear system) is given by $H^{0}\left(2 K_{\Sigma}\right)^{\mathbb{Z}_{5}}$ (resp. $\left.H^{0}\left(3 K_{\Sigma}\right)^{\mathbb{Z}_{5}}\right) . H^{0}\left(2 K_{\Sigma}\right)^{\mathbb{Z}_{5}}$ is generated by $x w, y z$, and $H^{0}\left(3 K_{\Sigma}\right)^{\mathbb{Z}_{5}}$ is generated by $x y^{2}, x^{2} z, y w^{2}, z^{2} w .\left|2 K_{\Sigma}\right|^{\mathbb{Z}_{5}}$ has 20 base points on $\Sigma$ :

$$
\left(1,0,-\epsilon^{i}, 0\right),\left(1,-\epsilon^{i}, 0,0\right),\left(0,-\epsilon^{i}, 0,1\right),\left(0,0,-\epsilon^{i}, 1\right) .
$$

Also, $\left|3 K_{\Sigma}\right|^{\mathbb{Z}_{5}}$ has 10 base points on $\Sigma$ : $\left(1,0,0,-\epsilon^{i}\right),\left(0,1,-\epsilon^{i}, 0\right)$.

The author proves the following three lemmas in [L2].

Lemma 1. Choose a generic element $X$ in the moduli space of Godeaux surfaces. Then $\left|2 K_{X}\right|$ has no base components, and has four simple base points. Let $p: S \rightarrow$ $X$ be the blow-up of four base points of $\left|2 K_{X}\right|$. Then each fiber of $f: S \rightarrow \mathbb{P}^{1}$ is a stable curve of genus four, and has at most one node.

Consider Deligne-Mumford compactification of the moduli space of curves of genus four, $\overline{\mathcal{M}_{4}}$. In this paper, $\overline{\mathcal{M}_{4}}$ is mostly a moduli functor instead of a moduli space, because we are usually interested in numerical data associated with the pull back to the pencil. The Hodge class $\lambda$ and the boundary classes $\delta_{0}, \delta_{1}, \delta_{2}$ are the standard basis of $\operatorname{Pic} \overline{\mathcal{M}_{4}} \otimes \mathbb{Q}[\mathrm{AC}] . f: S \rightarrow \mathbb{P}^{1}$ induces a morphism $\tilde{q}: \mathbb{P}^{1} \rightarrow \overline{\mathcal{M}}_{4}$. Then we obtain

$$
f_{*} \omega_{S / \mathbb{P}^{1}}=\mathcal{O}_{\mathbb{P}^{1}}(1)^{4} .
$$

Lemma 2. Let $f: S \rightarrow \mathbb{P}^{1}$ be the bicanonical pencil of a general Godeaux surface. Then $\lambda . \mathbb{P}^{1}=4, \delta_{0} \cdot \mathbb{P}^{1}=25, \delta_{1} \cdot \mathbb{P}^{1}=0$ and $\delta_{2} \cdot \mathbb{P}^{1}=2$.

Lemma 3. Let us call a stable curve $C$ of genus four a "Godeaux curve" if it occurs as a fiber of the bicanonical pencil for a smooth Godeaux surface. Then the family of Godeaux curves has five dimensions.

A general curve $C$ in the bicanonical pencil of a Godeaux surface is not a hyperelliptic curve [L1, §3.1]. In fact, there is no hyperelliptic curve in the bicanonical pencil of a Godeaux surface [L1, §4.3]. Let $X$ be a numerical Godeaux surface (surface of general type, with $p_{g}=q=0$, and $K^{2}=1$ ). Let $\tau_{i}$ be a nonzero torsion element of $H_{2}(X, \mathbb{Z})$. Then there is a unique effective divisor $C_{i}$ corresponding to $K_{X}+\tau_{i} . C_{i}, C_{j}$ intersect transversally at one point $P\left(C_{i}, C_{j}\right)$, by the following lemma of Reid in $\mathrm{Re}$.

Lemma 4. Let $X$ be a numerical Godeaux surface and $C_{i}=K_{X}+\tau_{i}$. Then

1. $h^{0}\left(K_{X}+\tau_{i}\right)=1$, 
2. $C_{i}, C_{j}$ intersect transversally at one point $P\left(C_{i}, C_{j}\right)$.

3. If $C_{i}, C_{j}, C_{k}$ are distinct, then $P\left(C_{i}, C_{j}\right)$ and $P\left(C_{i}, C_{k}\right)$ are distinct.

For a Godeaux surface $X, H_{2}(X, \mathbb{Z})_{t o r}=\mathbb{Z}_{5}$. So we have two special members of $\left|2 K_{X}\right|$, related to $\delta_{2}$. Write $D_{1}=C_{1}+C_{4}$ and $D_{2}=C_{2}+C_{3}$. Also denote

$$
\begin{aligned}
P_{1}=P\left(C_{1}, C_{2}\right), & P_{2}=P\left(C_{1}, C_{3}\right), \\
P_{3}=P\left(C_{4}, C_{2}\right), & P_{4}=P\left(C_{4}, C_{3}\right), \\
P=P\left(C_{1}, C_{4}\right), & Q=P\left(C_{2}, C_{3}\right) .
\end{aligned}
$$

Then $P_{1} \ldots P_{4}$ are base points of $\left|2 K_{X}\right|$ and $P, Q$ are base points of $\left|3 K_{X}\right|$.

Lemma 5. Let $C$ be a general member of the bicanonical pencil of a Godeaux surface. Then $K_{C}$ can be written as the combination of base points $P_{1} \ldots P_{4}$ in the following way:

$$
\begin{aligned}
K_{C} & =2 P_{1}+3 P_{2}+P_{4} \\
& =3 P_{1}+P_{2}+2 P_{3} \\
& =2 P_{2}+P_{3}+3 P_{4} \\
& =P_{1}+3 P_{3}+2 P_{4} .
\end{aligned}
$$

Also, we have $g_{3}^{1}$ and $h_{3}^{1}$ as the combination of base points, where $g_{3}^{1}+h_{3}^{1}=K_{C}$.

Proof. Since $K_{X}=2\left(K_{X}+\tau_{i}\right)-\left(K_{X}+\tau_{j}\right)$ where $2 \tau_{i}=\tau_{j}$, then we can write $\left.K_{X}\right|_{C}$ as the combination of base points.

$$
\begin{aligned}
\left.K_{X}\right|_{C} & =P_{1}+2 P_{2}-P_{3} \\
& =2 P_{1}+P_{3}-P_{4} \\
& =P_{2}+2 P_{4}-P_{1} \\
& =2 P_{3}+P_{4}-P_{2} .
\end{aligned}
$$

So $K_{C}=\left.3 K_{X}\right|_{C}=\left.2 K_{X}\right|_{C}+K_{X}\left|C=P_{1}+\cdots+P_{4}+K_{X}\right|_{C}$.

Since $2 P_{1}+P_{2} \sim\left(2 P_{3}+P_{4}-P_{2}\right)+\left(P_{4}-P_{3}\right)+P_{2}=P_{3}+2 P_{4}$, we have $2 P_{2}+P_{4} \sim P_{1}+2 P_{3}$, so

$$
\begin{aligned}
g_{3}^{1}: 2 P_{1}+P_{2} & \sim P_{3}+2 P_{4}, \\
h_{3}^{1}: 2 P_{2}+P_{4} & \sim P_{1}+2 P_{3}
\end{aligned}
$$

and their sum is $K_{C}$.

Theorem 6. The generic curve that is in the bicanonical pencil of a Godeaux surface can be constructed in the following way (see Figure 1.1).

Fix general four points $P_{1} \ldots P_{4}$ in $\mathbb{P}^{3}$. Draw four lines $L_{12}=\overline{P_{1} P_{2}}, L_{13}=\overline{P_{1} P_{3}}$, $L_{24}=\overline{P_{4} P_{2}}, L_{34}=\overline{P_{4} P_{3}}$, and consider the pencil of quadrics $\{Q\}$ containing these lines. Then consider the space $\{W\}$ of cubic surfaces in $\mathbb{P}^{3}$ which meet $L_{i j}$ doubly at $P_{i}$. The intersection curves $Q \cap W$ are shown in Figure 1.1. So $2 P_{1}+P_{2} \sim P_{3}+2 P_{4}$ gives $g_{3}^{1}$ and $P_{1}+2 P_{3} \sim 2 P_{2}+P_{4}$ gives $h_{3}^{1}$, and their sum is $K_{C}$.

Proof. According to Lemma 5, the curves related with Godeaux surfaces satisfy the condition of Theorem 6 . So we need to prove that the dimension of the family of curves satisfying the above condition is five.

Let $C$ be a canonical curve with genus four in $\mathbb{P}^{3}$. Let $\left(s_{1}, \ldots, s_{4}\right)$ be coordinates of $\mathbb{P}^{3}$ and $P_{1}=(1,0,0,0), P_{2}=(0,1,0,0), P_{3}=(0,0,1,0), P_{4}=(0,0,0,1)$. Since $C$ 


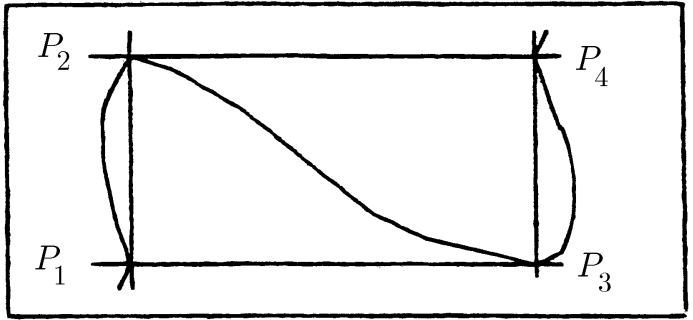

FiguRE 1.1. Generic curve in the bicanonical pencil

is a complete intersection of quadric $Q$ and cubic $F$, we have the following exact sequence and commutative diagrams, between the cohomology of tangent sheaf and the normal sheaf (see (1.2)).

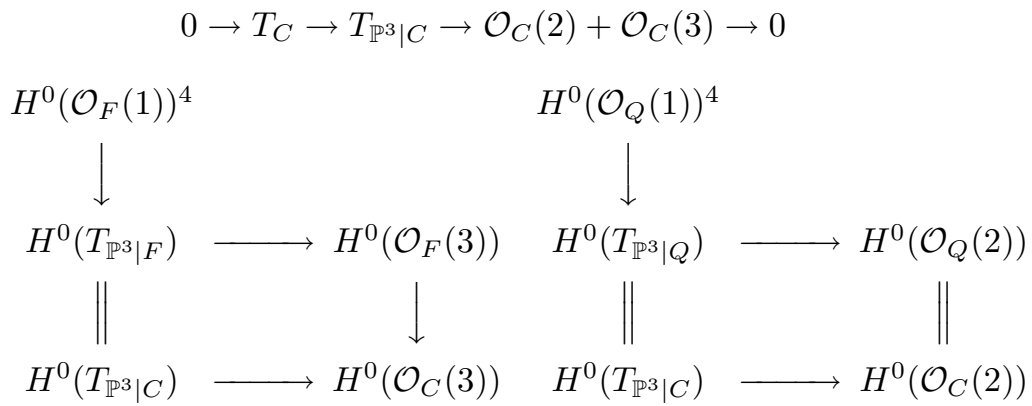

In (1.2), the composition of two maps from $H^{0}\left(\mathcal{O}_{F}(1)\right)^{4}$ to $H^{0}\left(\mathcal{O}_{F}(3)\right)$ and from $H^{0}\left(\mathcal{O}_{Q}(1)\right)^{4}$ to $H^{0}\left(\mathcal{O}_{Q}(2)\right)$ are the simple sum of $s_{i}$ times partial derivatives of $F$ and $Q$.

It is clear that the pencil of quadrics containing $\bigcup L_{i j}$ is generated by $s_{1} s_{4}, s_{2} s_{3}$. Fix one smooth quadric $Q$ in the above pencil, and consider the cubics containing $\bigcup L_{i j}$. These spaces are parameterized by the $\mathbb{P}^{7}$, generated by $s_{i} s_{1} s_{4}, s_{i} s_{2} s_{3}$ for $i=1 \ldots 4$. Then for given $Q$ and $W$, changing the cubic $W$ by an element of this $\mathbb{P}^{7}$ provides a trivial deformation of $C$ for the following reason.

Let $F$ be a cubic containing four lines,

$$
Q \cap F=(3,3)=(2,2)+(1,1)
$$

as a divisor in $Q$. Consider the following long exact sequence:

$$
0 \rightarrow H^{0}\left(T_{\mathbb{P}^{3} \mid C}\right) \rightarrow H^{0}\left(\mathcal{O}_{C}(2)\right)+H^{0}\left(\mathcal{O}_{C}(3)\right) \rightarrow H^{1}\left(T_{C}\right) \rightarrow .
$$

We may assume that $Q=s_{1} s_{4}+s_{2} s_{3}$. Then the partial derivative

$$
\left(\frac{\partial Q}{\partial s_{i}}\right)=\left(Q_{i}\right)=\left(s_{4}, s_{3}, s_{2}, s_{1}\right) .
$$

So the image of the map from $H^{0}\left(T_{\mathbb{P}^{3} \mid C}\right)$ to $H^{0}\left(\mathcal{O}_{C}(2)\right)+H^{0}\left(\mathcal{O}_{C}(3)\right)$ is generated by

$$
\left(\left.s_{i} s_{4}\right|_{C},\left.s_{i} F_{1}\right|_{C}\right),\left(\left.s_{i} s_{3}\right|_{C},\left.s_{i} F_{2}\right|_{C}\right),\left(\left.s_{i} s_{2}\right|_{C},\left.s_{i} F_{3}\right|_{C}\right),\left(\left.s_{i} s_{1}\right|_{C},\left.s_{i} F_{4}\right|_{C}\right)
$$

where $F_{i}=\left.\frac{\partial F}{\partial s_{i}} \cdot s_{1} s_{4}\right|_{C}=\left.s_{2} s_{3}\right|_{C}=3\left(P_{1}+\cdots+P_{4}\right)$. Therefore there is a fourdimensional subspace of $H^{0}\left(T_{\mathbb{P}^{3} \mid C}\right)$ which fixes $H^{0}\left(\mathcal{O}_{C}\left(3 P_{1}+\cdots+3 P_{4}\right)\right)$. When we fix $Q$, and deform $Q \cap W$ by changing $W$ in the direction of cubics containing four 
lines, we obtain the subspace $H^{0}\left(\mathcal{O}_{C}\left(3 P_{1}+\cdots+3 P_{4}+q_{1}+\cdots+q_{6}\right)\right.$ of $H^{0}\left(\mathcal{O}_{C}(3)\right)$, varied only by $q_{1} \ldots q_{6}$. That is the same as $H^{0}\left(\mathcal{O}_{C}\left(K_{C}\right)\right)$. So the image of the fourdimensional subspace of $H^{0}\left(T_{\mathbb{P}^{3} \mid C}\right)$ goes surjectively to $H^{0}\left(\mathcal{O}_{C}\left(K_{C}\right)\right)$. Therefore there is no deformation of $C$ by varying $W$ by an element of the space of cubics containing four lines.

Then $g_{3}^{1}, h_{3}^{1}$, the tangent conditions at $P_{i}$ give a 13-dimensional projective space of cubics. In this case four-tangent conditions do not give eight conditions, they give seven conditions by $g_{3}^{1}, h_{3}^{1}$ (four points and three tangent directions determine the fourth tangent direction). So for fixing $Q$, we have a four-dimensional deformation of $H^{1}\left(T_{C}\right)$ by changing of cubics. By changing the quadrics in the pencil, we have a five-dimensional family of curves of genus four.

\section{The associated Prym Curves}

By Lemma 3, each curve in this five-dimensional family of Godeaux curves occurs in a four-dimensional family of Godeaux surfaces (because Godeaux surfaces have eight moduli). The author finds a four-dimensional subfamily of Godeaux surfaces on which each of these curves occurs with finite frequency in [L1 \$3.2], namely Godeaux surfaces coming from $\mathbb{Z}_{5}$-invariant symmetric determinantal quintics in $\mathbb{P}^{3}$. A general symmetric determinantal quintic has an even set of twenty nodes C1 (an even set of nodes means that there is a double cover branched of only those nodal points).

Each determinantal Godeaux surface $X$ has a double covering. The four fixed points of a $\mathbb{Z}_{2}$-action are exactly the four nodes of $X$. Since these four nodes are not four base points of $\left|2 K_{X}\right|$, the general member $C$ of $\left|2 K_{X}\right|$ has a double covering $\tilde{C}$ without fixed points. So then it is possible to construct a "Prym curve" $D$ for $(\tilde{C}, C)[\mathrm{Mu}$. The Prym curve $D$ associated to $(\tilde{C}, C)$ is a curve of genus three with two distinguished linear series $g_{4}^{1}, h_{4}^{1}$ (associated to the two $g_{3}^{1}$ 's on $C$ ) for which $g_{4}^{1}+h_{4}^{1}=2 K_{D}$. We characterized this subfamily of curves in $\overline{\mathcal{M}}_{4}$ in Theorem 6 by using the base points of $\left|2 K_{X}\right|$. In this section, we will characterize the subfamily of Prym curves in $\overline{\mathcal{M}_{3}}$ generated by this construction. Also we can construct reversely. These constructions are given in $[\mathrm{Rc}]$.

Let $C$ be a curve of genus four with a $g_{3}^{1}$ and $\sigma: \tilde{C} \rightarrow C$ an unbranched double cover, then the $2^{3}$ liftings of $P+Q+R \in g_{3}^{1}$ to a divisor of degree three on $\tilde{C}$ break up into two components according to the parity of how many come from the "top" sheet. This induces a curve $\tilde{D}$ in $\tilde{C}^{(3)}$. The involution on $\tilde{D}$ coming from parity gives a curve $D$ of genus three and $\tilde{D} \rightarrow D$ is the reducible unbranched double covering. Also $D$ has its natural $g_{4}^{1}$ coming from the four liftings. Conversely, let $D$ be a curve of genus three with a $g_{4}^{1}$. Then let $\tilde{C}$ be the set of pairs $\{p, q\}$ on $D$ such that there exist $r, s$ on $D$ with $p+q+r+s \in g_{4}^{1}$. Then $\tilde{C}$ has a natural involution $\{p, q\} \Leftrightarrow\{r, s\}$, and quotient $C$ has genus four with a $g_{3}^{1}$, we have

$$
\operatorname{Jac}(D)=\operatorname{Prym}(\tilde{C}, C) .
$$

The following theorem is due to Donagi in [Do].

Theorem 7. The above construction gives a birational morphism between trigonal curves $C$ of genus $g$ with a double cover $\tilde{C}$ and tetragonal curves $D$ of genus $g-1$.

Consider $C$ and $g_{3}^{1}, h_{3}^{1}$ in Lemma 5 .

$$
g_{3}^{1}: 2 P_{1}+P_{2} \sim P_{3}+2 P_{4},
$$




$$
h_{3}^{1}: 2 P_{2}+P_{4} \sim P_{1}+2 P_{3}
$$

and their sum is $K_{C}$. Let $P_{1}^{1}, P_{1}^{2}, \ldots P_{4}^{1}, P_{4}^{2}$ denote the liftings of $P_{1}, P_{2}, P_{3}, P_{4}$ in $\tilde{C}$.

For convenience let us assume that the odd parity induces the curve $D$. We denote the points of $D$ built from these lifting as follows:

$$
\begin{array}{ll}
\left(P_{1}^{1}, P_{1}^{1}, P_{2}^{1}\right) \rightarrow y_{1}, & \left(P_{2}^{1}, P_{2}^{1}, P_{4}^{1}\right) \rightarrow y_{2}, \\
\left(P_{1}^{1}, P_{1}^{2}, P_{2}^{2}\right) \rightarrow x_{1}, & \left(P_{2}^{1}, P_{2}^{2}, P_{4}^{2}\right) \rightarrow x_{2}, \\
\left(P_{1}^{2}, P_{1}^{2}, P_{2}^{1}\right) \rightarrow z_{1}, & \left(P_{2}^{2}, P_{2}^{2}, P_{4}^{1}\right) \rightarrow z_{2}, \\
\left(P_{4}^{1}, P_{4}^{1}, P_{3}^{1}\right) \rightarrow y_{4}, \quad\left(P_{3}^{1}, P_{3}^{1}, P_{1}^{1}\right) \rightarrow y_{3}, \\
\left(P_{4}^{1}, P_{4}^{2}, P_{3}^{2}\right) \rightarrow x_{4}, \quad\left(P_{3}^{1}, P_{3}^{2}, P_{1}^{2}\right) \rightarrow x_{3}, \\
\left(P_{4}^{2}, P_{4}^{2}, P_{3}^{1}\right) \rightarrow z_{4}, \quad\left(P_{3}^{2}, P_{3}^{2}, P_{1}^{1}\right) \rightarrow z_{3} .
\end{array}
$$

Denote $D$ to be a canonical curve of genus three, i.e., smooth quartic plane curve. Then all $g_{4}^{1}$ 's can be understood in the following way. Consider four fixed points in $D$ and a pencil of conics through these four fixed points. Then the other four intersection points give a $g_{4}^{1}$. By the bijectiveness of Theorem 7 and duality, we have $g_{4}^{1}, h_{4}^{1}=2 K_{D}-g_{4}^{1}$ on $D$ such that

$$
\begin{aligned}
g_{4}^{1}: 2 x_{1}+y_{1}+z_{1} & \sim 2 x_{4}+y_{4}+z_{4}, \\
h_{4}^{1}: 2 x_{2}+y_{2}+z_{2} & \sim 2 x_{3}+y_{3}+z_{3} .
\end{aligned}
$$

And we have four conics $C_{12}, C_{13}, C_{24}, C_{34}$ :

$$
\begin{aligned}
& \left.C_{12}\right|_{D}: 2 x_{1}+y_{1}+z_{1}+2 x_{2}+y_{2}+z_{2}, \\
& \left.C_{13}\right|_{D}: 2 x_{1}+y_{1}+z_{1}+2 x_{3}+y_{3}+z_{3}, \\
& \left.C_{24}\right|_{D}: 2 x_{2}+y_{2}+z_{2}+2 x_{4}+y_{4}+z_{4}, \\
& \left.C_{34}\right|_{D}: 2 x_{3}+y_{3}+z_{3}+2 x_{4}+y_{4}+z_{4} .
\end{aligned}
$$

There is a natural map from $D^{(2)}$ to $D^{(2)}$ by $K_{D}-x-y$ for $\{x, y\} \in D^{(2)}$. Consider two constructions $\tilde{C}_{g}$ in $D^{(2)}$ using $g_{4}^{1}$ and $\tilde{C}_{h}$ using $h_{4}^{1}$. Taking the quotients by the respective involutions we have curves $C_{g}$ and $C_{h}$. The natural map $x+y \rightarrow K_{D}-x-y$ gives an isomorphism between $C_{g}$ and $C_{h}$. Working out this correspondence explicitly with respect to the points $P_{i}^{j}$ above, we conclude that $\overline{x_{3} z_{3}}$ passes through $x_{1}, \overline{x_{1} z_{1}}$ through $x_{2}, \overline{x_{4} z_{4}}$ through $x_{3}, \overline{x_{2} z_{2}}$ through $x_{4}$. Then this property and $2 K_{D}=g_{4}^{1}+h_{4}^{1}$ gives that each conic $C_{i j}$ is the union of two lines,

$$
\begin{aligned}
& C_{12}=\overline{x_{1} x_{2}}+\overline{x_{1} z_{2}}, C_{34}=\overline{x_{3} x_{4}}+\overline{x_{4} z_{3}}, \\
& C_{24}=\overline{x_{2} x_{4}}+\overline{x_{2} z_{4}}, C_{13}=\overline{x_{1} x_{3}}+\overline{x_{3} z_{1}} .
\end{aligned}
$$

This induces the following theorem.

Theorem 8. The family of Prym curves of determinantal Godeaux surfaces can be constructed in the following way. Fix four points $\left\{x_{i}\right\}$ and four lines in $\mathbb{P}^{2}$ as shown in Figure 2.1.

On each line pick a point $\left\{z_{i}\right\}$. Consider four conics $C_{12}, C_{34}, C_{24}, C_{13}$ as above. Then consider the pencil of quartics,

$$
\lambda\left(C_{12} C_{34}\right)+\mu\left(C_{13} C_{24}\right)
$$




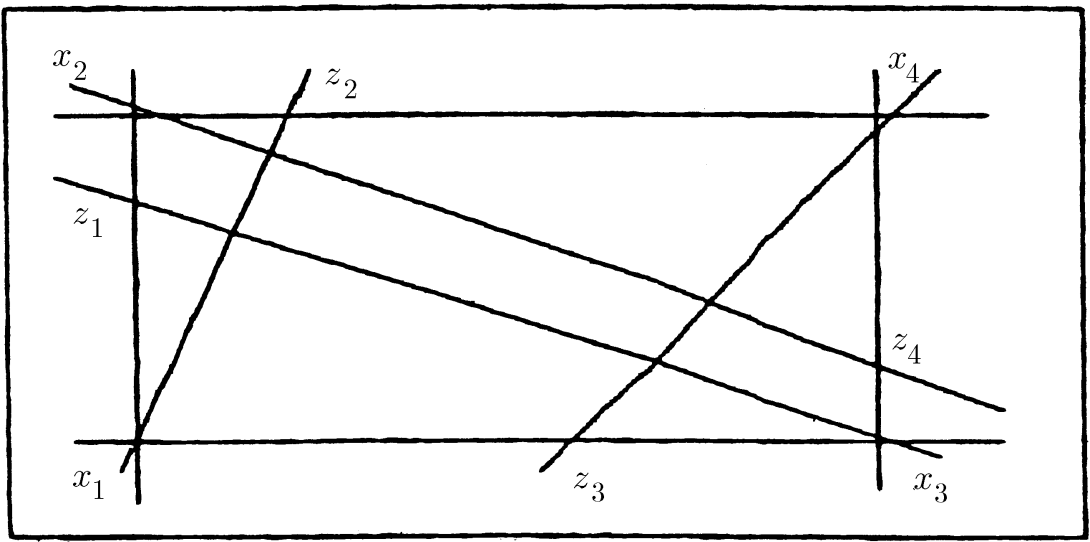

Figure 2.1. Associated Prym curves

The set of all such quartic plane curves, obtained by moving $z_{i}$ on the line, forms a five-dimensional family.

Proof. Figure 2.1 determines four points $y_{1}, \ldots y_{4}$ such that $y_{1} \in \overline{x_{1} z_{2}} \cap \overline{x_{3} z_{1}}$, $y_{2} \in \overline{x_{1} z_{2}} \cap \overline{x_{2} z_{4}}, y_{3} \in \overline{x_{4} z_{3}} \cap \overline{x_{3} z_{1}}, y_{4} \in \overline{x_{4} z_{3}} \cap \overline{x_{2} z_{4}}$.

Then $2 x_{1}+y_{1}+z_{1} \sim 2 x_{4}+y_{4}+z_{4}, 2 x_{2}+y_{2}+z_{2} \sim 2 x_{3}+y_{3}+z_{3}$ are the elements of $g_{4}^{1}, h_{4}^{1}$ in Prym curve $D$. We show that these $g_{4}^{1}, h_{4}^{1}$ correspond to the elements of $g_{3}^{1}$ and $h_{3}^{1}$ in $C$ (Theorem 6) for the determinantal Godeaux surfaces. Since the family of determinantal Godeaux curves has five dimensions and the family of curves constructed above has no more than five dimensions, we obtain Theorem 8.

\section{The Reverse COnStruction}

Consider the linear system of conics in $\mathbb{P}^{2}$. Let $\Pi$ be a general three-dimensional linear subspace of $\mathbb{P}\left(H^{0}\left(\mathbb{P}^{2}, \mathcal{O}_{\mathbb{P}^{2}}(2)\right)\right) \cong \mathbb{P}^{5}$. Let $V_{4}$ denote the set of conics in $\mathbb{P}^{2}$ of rank $\leq 2$ (two lines). $V_{4}$ is an irreducible variety of dimension 4 and degree 3 . Let $V_{2}$ denote the set of conics in $\mathbb{P}^{2}$ of rank $\leq 1$ (double line). $V_{2}$ is an irreducible variety of dimension 2 and degree 4 . Then $\Pi \cap V_{4}$ is a cubic surface with four nodes (Segre cubic surface). This cubic surface has a natural double covering that is branched over the four nodal points.

Consider four double lines in $\mathbb{P}^{2}$ (Figure 2.1): $2 \overline{x_{1} x_{2}}, 2 \overline{x_{1} x_{3}}, 2 \overline{x_{2} x_{4}}, 2 \overline{x_{3} x_{4}}$. These four double lines give four points $P_{12}=P_{21}, P_{13}=P_{31}, P_{24}=P_{42}, P_{34}=P_{43}$ in $V_{2}$ and determine $\Pi=\mathbb{P}^{3}$ in $\mathbb{P}^{5}$. Also these four points are exactly the node points of Segre cubic $S_{0}=\Pi \cap V_{4}$.

Consider a pencil of conics (union of two lines) in Figure 2.1, $\overline{x_{1} x_{2}} \cup \overline{x_{1} z_{2}}$ given by moving of $z_{2}$ and fixing of $x_{1}, x_{2}$. This gives a line that connects $P_{12}, P_{13}$. By the same construction of the other three lines, we have a configuration of four lines and four points. The quartic curve in the associated pencil of Prym curves determined by $z_{1}, z_{2}, z_{3}, z_{4}$ is given by $\lambda\left(C_{12} C_{34}\right)+\mu\left(C_{13} C_{24}\right)$ for some $(\lambda, \mu) \in \mathbb{P}^{1}$ (see Theorem 8). These four points $z_{i}$ determine four points $P_{1}, P_{2}, P_{3}, P_{4}$ where $P_{i}$ is on the line $\overline{P_{i j} P_{i k}}$. Then draw four lines,

$$
\overline{P_{1} P_{2}}, \overline{P_{1} P_{3}}, \overline{P_{2} P_{4}}, \overline{P_{3} P_{4}}
$$




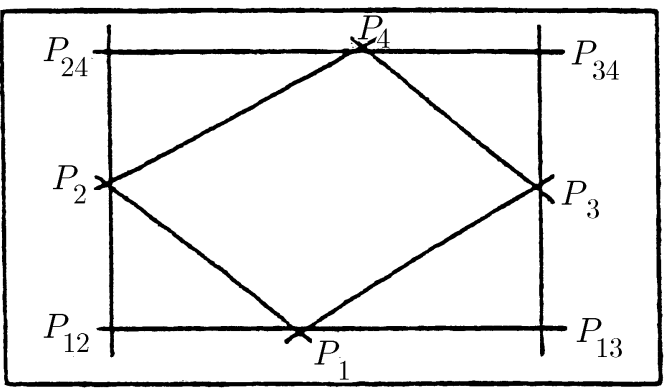

FiguRE 3.1. reverse construction in Segre cubic surface

(see Figure 3.1).

Choose the quadric of the pencil containing the four lines $\overline{P_{i} P_{j}}$ which is given by $(\lambda, \mu)$. Then $Q \cap S_{0}$ gives the reverse construction of the genus four curve. Consider now the quadric linear system in $\mathbb{P}^{3}$ :

$$
\mathbb{P}\left(H^{0}\left(\mathbb{P}^{3}, \mathcal{O}(2)\right)\right)=\mathbb{P}^{9} .
$$

Inside of the bicanonical pencil of a Godeaux surface or a determinantal Godeaux surface there are two special quadrics $x w, y z$. The general quadric associated to a bicanonical pencil lies in

$$
s\left(\lambda_{1} x+\lambda_{2} y\right)\left(\lambda_{3} z+\lambda_{4} w\right)+t\left(\mu_{1} x+\mu_{2} z\right)\left(\mu_{3} y+\mu_{4} w\right) .
$$

Therefore we have a morphism

$$
\varphi: \mathbb{P}^{1} \rightarrow \mathbb{P}^{5} \subset \mathbb{P}^{9}
$$

where $\mathbb{P}^{5}$ is given by $x z, x w, y z, y w, x y, z w$. Also we have a genus four fibration $\mathcal{V}$ over $\mathbb{P}^{5}$, whose fiber at $x \in \mathbb{P}^{5}$ is $S_{0} \cap Q_{x}$. So we have the following commutative diagram:

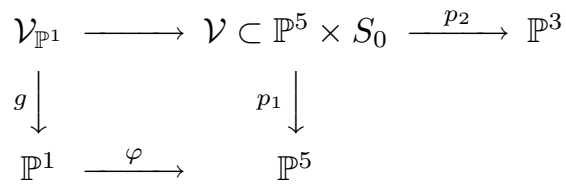

Lemma 9. Let $S_{0}$ be a Segre cubic surface and $\ell$ a line through two nodes. Then the rank of the Hessian matrix at a generic point in the line is two.

Proof. Let $(x, y, z, w)$ be the coordinates of $\mathbb{P}^{3}$. Then the equation of Segre cubic is the determinant of

$$
\left(\begin{array}{lll}
x & 0 & 0 \\
0 & y & 0 \\
0 & 0 & z
\end{array}\right)+w\left(\begin{array}{lll}
1 & 1 & 1 \\
1 & 1 & 1 \\
1 & 1 & 1
\end{array}\right) .
$$

Set $w=1$ and choose the affine coordinates $(x, y, z)$. The four nodal points are

$$
(0,0,0),(1,0,0),(0,1,0),(0,0,1)
$$

and the intersection at $x=0$ is $y z=0$. In the affine coordinate $(w=1)$ quadric: $x+\lambda y z$ for degree of $\lambda y z=3$, cubic $\left.\right|_{x=0}=y z$.

So the Hessian of the intersection has rank two.

Theorem 10. Degree of $\varphi=3$. 
Proof. Theorem 10 can be proved by an adjunction formula. Since $K_{\mathbb{P}^{5} \times \mathbb{P}^{3}}=$ $p_{1}^{*} \mathcal{O}_{\mathbb{P}^{5}}(-6) \otimes p_{2}^{*} \mathcal{O}_{\mathbb{P}^{3}}(-4)$, so we have

$$
K_{\mathbb{P}^{5} \times S_{0}}=p_{1}^{*} \mathcal{O}_{\mathbb{P}^{5}}(-6) \otimes p_{2}^{*} \mathcal{O}_{S_{0}}(-1) .
$$

Let $N$ be the normal bundle of $\mathbb{P}^{1}$ in $\mathbb{P}^{5}$, and $d$ the degree of $\varphi$. Then

$$
\left.\mathcal{O}_{\mathbb{P} 5}(-6) \otimes \operatorname{det} N\right|_{\mathbb{P}^{1}}=-2,
$$

we have $\operatorname{det} N=6 d-2$ and

$$
K \mathcal{V}_{\mathbb{P}^{1}}=p_{1}^{*} \mathcal{O}_{\mathbb{P}^{1}}(d-2) \otimes p_{2}^{*} \mathcal{O}_{S_{0}}(1) .
$$

So $g_{*} K_{\mathcal{V}_{\mathbb{P} 1}}=\mathcal{O}_{\mathbb{P}^{1}}(d-2)^{4}$, and $g_{*} K_{\mathcal{V}_{\mathbb{p} 1} / \mathbb{P}^{1}}=\mathcal{O}_{\mathbb{P}^{1}}(d)^{4}$. Let $p: S \rightarrow X$ be a blow-up of four base points of the bicanonical pencil and $p^{\prime}: S^{\prime} \rightarrow S$ denote a blow-up of the two base points of $p^{*}\left(3 K_{X}\right)$. Consider the following diagram.

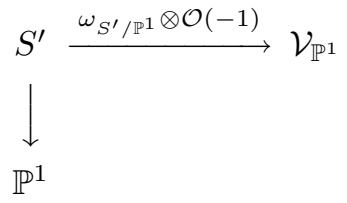

Because we have two multiple fibers with multiplicity two in $\mathcal{V}_{\mathbb{P}^{1}} \rightarrow \mathbb{P}^{1}$ and by Lemma 9 , the tangent cone through the line is a generically normal crossing, so the relative dualizing sheaf of the normalization is the pull back of the relative dualizing sheaf tensored with the ideal sheaf of the two special fibers of the Godeaux surface. Therefore $g_{*} K_{\mathcal{V}^{1} / \mathbb{P}^{1}} \otimes \mathcal{O}_{\mathbb{P}^{1}}(-2)=\mathcal{O}_{\mathbb{P}^{1}}(1)^{4}$, so $d=3$.

According to Theorem 10, the degree of the map from the bicanonical pencil to each line $\overline{P_{i j} P_{i k}}$ is one, i.e.

$$
K_{\mathcal{V}}=\left.p_{1}^{*} \mathcal{O}_{\mathbb{P}^{5}}(-5) \otimes p_{2}^{*} \mathcal{O}_{\mathbb{P}^{3}}(1)\right|_{\mathcal{V}} .
$$

This implies that the degree of the map from the pencil of associated Prym curves, related with a determinantal Godeaux surface, to each of the four lines

$\left(\overline{x_{1} x_{2}}, \overline{x_{1} x_{3}}, \overline{x_{4} x_{2}}, \overline{x_{4} x_{3}}\right)$

is one (see Figure 2.1).

Remark. The family of twisted cubics in $\mathbb{P}^{5}$ through the two marked points, $x w, y z$, is via projection from these points, a bundle over the Grassmannian variety $\mathcal{G}$ of lines in $\mathbb{P}^{3}$. In affine coordinates the general twisted cubic coming from a determinantal Godeaux surface is of the form

$$
\left(x+c_{1} t y\right)\left(c_{2} t z+w\right)+t\left(x+c_{3} t z\right)\left(c_{4} t y+w\right)
$$

From this it is easy to check that these twisted cubics fiber over

$$
H=\overline{\{x z, y w\}} \times \overline{\{x y, z w\}}=\mathbb{P}^{1} \times \mathbb{P}^{1} \subseteq \mathcal{G} \subseteq \mathbb{P}^{5}
$$

where $H$ is just the intersection of two tangent hyperplane sections of $\mathcal{G}$. Thus the problem of compactifying the space of "Godeaux twisted cubics" is reduced to studying the compactification of the following space:

Let $p=\{x w\}, q=\{y z\}, p^{\prime}=\left\{c_{2} x z+c_{1} y w\right\},=q^{\prime}=\left\{c_{4} x y+c_{3} z w\right\}$. Let $L=\overline{p p^{\prime}}$, $M=\overline{q q^{\prime}}$. We compactify the surface of twisted cubics in $\mathbb{P}^{3}$ tangent to $L$ at $p$ and tangent to $M$ at $q$ with osculating plane at $p$ given by $\left\langle p, p^{\prime}, q^{\prime}\right\rangle$ and osculating plane at $q$ given by $\left\langle q, q^{\prime}, p^{\prime}\right\rangle$. 
This compactification is worked out in [PS]. The associated relative dualizing sheaf can be computed easily. So if we let $\mathcal{S}^{*} \rightarrow \Delta^{*} \times \mathbb{P}^{1}$ be a family of four point blow-ups of determinantal Godeaux surfaces over the punctured disk, this family determines an associated family $\mathcal{T}^{*}$ of canonical curves

$$
\mathcal{T}^{*} \subseteq \Delta^{*} \times \mathbb{P}^{1} \times S_{0} \subseteq \Delta^{*} \times \mathbb{P}^{1} \times \mathbb{P}^{3}
$$

The relative dualizing sheaf of $\mathcal{T}^{*}$ over $\Delta^{*}$ is

$$
\mathcal{O}_{\mathbb{P}^{1}}(3) \otimes \mathcal{O}_{\mathbb{P}^{3}}(1)
$$

by Theorem 10, and by Lemma 9 , the relative dualizing sheaf is

$$
\mathcal{O}_{\mathbb{P}^{1}}(1) \otimes \mathcal{O}_{\mathbb{P}^{3}}(1) .
$$

Then we compactify $\Delta^{*} \times \mathbb{P}^{1}$ in the above space of twisted cubics and use the relative dualizing sheaf to compute the dualizing sheaf of the compactified families

$$
\mathcal{S} \rightarrow \mathcal{T} \rightarrow \Delta \text {. }
$$

We intend to study this compactification in detail in a future work.

\section{REFERENCES}

[AC] E. Arbarello and M. Cornalba, The Picard groups of the moduli spaces of curves, Topology 26 (1987), 153-171. MR 88e:14032

[C1] F. Catanese, Babbage's conjecture, contact of surfaces, symmetric determinantal varieties and applications, Invent. Math. 63 (1981), 433-465. MR 83c:14026

[C2] F. Catanese, On the moduli spaces of surfaces of general type, J. Diff. Geom. 19 (1984), 483-515. MR 86h:14031

[Do] R. Donagi, The fibers of the Prym map, Contemp. Math. 136 (1992), 55-125. MR 94e:14037

[L1] Y. Lee, Degeneration of numerical Godeaux surfaces, Ph.D. Thesis, University of Utah (1997).

[L2] Y. Lee, Bicanonical pencil of a determinantal Barlow surface, Trans. Amer. Math. Soc., to appear. CMP 99:17

[Mi] Y. Miyaoka, Tricanonical maps of numerical Godeaux surfaces, Invent. Math. 34 (1976), 99-111. MR 53:13236

[Mu] D. Mumford, Prym varieties. I, Contributions to Analysis (L. V. Ahlfors, I. Kra, B. Maskit, L. Nirenberg, eds.), Academic Press, New York, 1974, pp. 325-350. MR 52:415

[PS] R. Piene and M. Schlessinger, The Hilbert scheme compactification of the space of twisted cubics, Amer. J. Math. 107 (1985), 761-774. MR 86m:14004]

[Rc] S. Recillas, Jacobians of curves with a $g_{4}^{1}$ are the Prym's of trigonal curves, Bol. Soc. Math. Mexicana 19 (1974), 9-13. MR 58:666

[Re] M. Reid, Surfaces with $p_{g}=0, K^{2}=1$, J. Fac. Sci. Univ. of Tokyo 25 (1978), 75-92. MR 80h:14018

Korea Institute for Advanced Study, 207-43 Cheongryangri-dong, Dongdaemun-gu, SEOUl 130-012, Korea

Current address: Department of Mathematics, Sogang University, Sinsu-dong, Mapo-gu, Seoul 121-742, Korea

E-mail address: ynlee@ccs.sogang.ac.kr 